\title{
Bifunctional Metal Phosphide FeMnP Films from Single Source Metal Organic Chemical Vapor Deposition for Efficient Overall Water Splitting
}

Zhenhuan Zhao ${ }^{a c \dagger}$, Desmond E. Schipper ${ }^{b \dagger}$, Andrew P. Leitner ${ }^{b}$, Hari Thirumalai ${ }^{d}$, Jing-Han Chen ${ }^{b}$, Lixin Xie ${ }^{e}$, Fan Qin $^{c}$, Md Kamrul Alam ${ }^{c}$, Lars C. Grabow ${ }^{d}$, Shuo Chen $^{e}$, Dezhi Wang ${ }^{e}$, Zhifeng Ren ${ }^{e}$, Zhiming Wang ${ }^{a^{*}}$, Kenton H. Whitmire ${ }^{b^{*}}$ and Jiming Bao ${ }^{c a^{*}}$

${ }^{a}$ Institute of Fundamental and Frontier Sciences, University of Electronic Science and Technology of China, Chengdu 610054, China

${ }^{b}$ Department of Chemistry, Rice University, Houston, Texas 77005, United States

${ }^{c}$ Department of Electrical and Computer Engineering, University of Houston, Houston, Texas 77204, United States

${ }^{d}$ Department of Chemical and Biomolecular Engineering, University of Houston, Houston, Texas 77204, United States

${ }^{e}$ Department of Physics \& Texas Center for Superconductivity of the University of Houston, University of Houston, Houston, Texas 77204, United States

${ }^{\dagger}$ Authors contributed equally

"Corresponding authors: zhmwang@gmail.com,whitmir@rice.edu,jbao@uh.edu 
Abstract

Developing stable and efficient bifunctional catalysts for overall water splitting into hydrogen and oxygen is a critical step in the realization of several clean-energy technologies. Here we report a robust and highly active electrocatalyst that is constructed by deposition of the ternary metal phosphide FeMnP onto graphene-protected nickel foam by metal-organic chemical vapor deposition from a single source precursor. FeMnP exhibits high electrocatalytic activity toward both the hydrogen evolution reaction (HER) and the oxygen evolution reaction (OER). Utilizing FeMnP/GNF as both the anode and the cathode for overall water splitting, a current density of $10 \mathrm{~mA} \mathrm{~cm}$ ch $^{-2}$ is achieved at a cell voltage of as low as $1.55 \mathrm{~V}$ with excellent stability. Complementary density functional theory (DFT) calculations suggest that facets exposing both Fe and Mn sites are necessary to achieve high HER activity. The present work provides a facile strategy for fabricating highly efficient electrocatalysts from earth-abundant materials for overall water splitting.

Keywords: FeMnP, MOCVD, bifunctional electrocatalyst, overall water splitting 


\section{Introduction}

The electrolysis of water into hydrogen fuel and oxygen offers a convenient route to store intermittent solar and wind energy chemically and an ideal solution for channelling off-peak power production and minimizing energy losses incurred in power transmission from often remote locations [1]. The utilization of active, stable electrocatalysts with lower overpotentials will increase efficiency and stability and enable commercialization $[2,3]$. Over the past several decades, tremendous progress has been made in the development of highly active catalysts composed of earth-abundant elements including transition metal phosphides (TMPs) [4-6], carbides [7-9], nitrides [10-12], and chalcogenides [13-15] as well as carbon-based nanomaterials [16-18] for OER and HER. Catalysts active for both reactions in the same electrolyte are preferred for practical applications. However, the vast majority of existing catalysts are unsuitable for use in the same electrolyte due to the mismatch of $\mathrm{pH}$ ranges in which the electrocatalysts are both stable and sufficiently active.

Since the first report of the TMP $\mathrm{Ni}_{2} \mathrm{P}$ as an electrocatalyst for water splitting in 2013 [19], TMPs have emerged as premier electrocatalysts for OER [20, 21], HER [4, 19, 22, 23], and in some cases overall water splitting [24-26]. For the TMPs that are capable of overall water splitting, their bifunctionality arises from the respective TMP's ability to catalyse the HER and serve as a precatalyst for the OER [27]. The current preparation methods for TMPs can be grouped into four main routes: thermal phosphidation of films [28] or nanostructured/bulk alloys [20, 26], electrochemical deposition [29], metallurgical synthesis [30], and solvothermal methods [22, 23]. 
However, these methods require harsh conditions with toxic gaseous chemicals, and offer little control over the metal-phosphorus stoichiometry, phase purity, and conductivity. For example, while $\left(\mathrm{Co}_{0.52} \mathrm{Fe}_{0.48}\right)_{2} \mathrm{P}$ can be used as both anode and cathode to achieve a low cell voltage of $1.53 \mathrm{~V}$, the material fabrication process, which involves a combination of arc-melting $\mathrm{Co}_{2} \mathrm{P}, \mathrm{Co}$ and $\mathrm{Fe}$ followed by selective electrochemical etching [26], is not suitable for scaled-up production. As TMPs are known to oxidize rapidly to highly active metal oxyhydroxides at their surface upon OER operation, it is therefore important to retain a conductive TMP core for fast charge transport, a structural feature which is highly dependent on the fabrication method. With several examples of bifunctional TMPs, there is now an impetus for the development of new strategies to fabricate these high performance materials.

The deposition of the TMP by metal-organic chemical vapor deposition (MOCVD) holds promise as a scalable methodology by which binder-free electrodes can be fabricated. Recently, it has been demonstrated that mixed-metal carbonyl complexes can serve as volatile, stoichiometry-controlled single-source precursors (SSPs) for MOCVD at mild temperatures [31-33]. With this method, binary and ternary TMPs can be prepared by tailoring the metal elements in a single volatile precursor or by simply blending isolobal organometallic compound precursors. Recently, the SSP-MOCVD method was used to grow FeMnP on $\mathrm{TiO}_{2}$-nanorod arrays for photoelectrochemical OER, and it was found that the $\mathrm{TiO}_{2} / \mathrm{FeMnP}$ core/shell structure remained stable at the theoretical photocurrent density of $\mathrm{TiO}_{2}[34,35]$. In the present work, we used the SSP-MOCVD to grow nanostructured ternary FeMnP from the 
volatile precursor $\mathrm{FeMn}(\mathrm{CO})_{8}\left(\mu-\mathrm{PH}_{2}\right)$ [36] onto nickel foam (NF) and graphene-protected nickel foam (GNF), and demonstrated FeMnP as a bifunctional catalyst for efficient and stable overall water splitting. Here, the SSP-MOCVD for the synthesis of a TMP on a conductive substrate is presented as an effective route to meeting the necessity of stable, high surface area structures with metallic cores for catalysis.

\section{Results and discussion}

The organometallic precursor $\mathrm{FeMn}(\mathrm{CO})_{8}\left(\mu-\mathrm{PH}_{2}\right)$ was synthesized and used as the single-source precursor for the deposition of FeMnP nanoplatelets on both nickel foam (NF) and graphene-coated nickel foam (GNF) using a homemade MOCVD setup (Fig. S1) [32, 36]. The high quality of the CVD grown GNF was confirmed by the negligible D band in its Raman spectra, as shown in Fig. S2. The 2D to G ratios show the coexistence of monolayer graphene and multilayer graphene on nickel foam $[37,38]$. The morphologies of FeMnP on NF and GNF were observed with scanning electron microscopy (SEM). Before the deposition, they both possessed smooth surfaces (Fig. S3). SEM images at lower magnification show that both the NF and GNF were uniformly covered by FeMnP nanoplatelets with a film thickness of about $5 \mu \mathrm{m}$ (Fig. S4). Closer observation at higher magnification shows that FeMnP on NF and GNF have similar platelet-like structures with thicknesses of about $50 \mathrm{~nm}$ (Fig. $1 \mathrm{~A}$ and $\mathrm{B})$. The X-ray diffraction (XRD) pattern shows two diffraction peaks located at $40^{\circ}$ and $42^{\circ} 2 \theta$ (Fig. S5), which can be indexed to the (111) and (201) plane of the 
hexagonal FeMnP phase according to the PDF card (04-006-1275) refined with GSAS software to yield lattice constants of $a=b=5.923 \AA$ and $c=3.527 \AA[39,40]$. The diffraction peaks at $44.5^{\circ}, 51.8^{\circ}$ and $76.4^{\circ} 2 \theta$ are assigned to (111), (200) and (220) planes of NF (JCPDS no. 65-2865) [15].
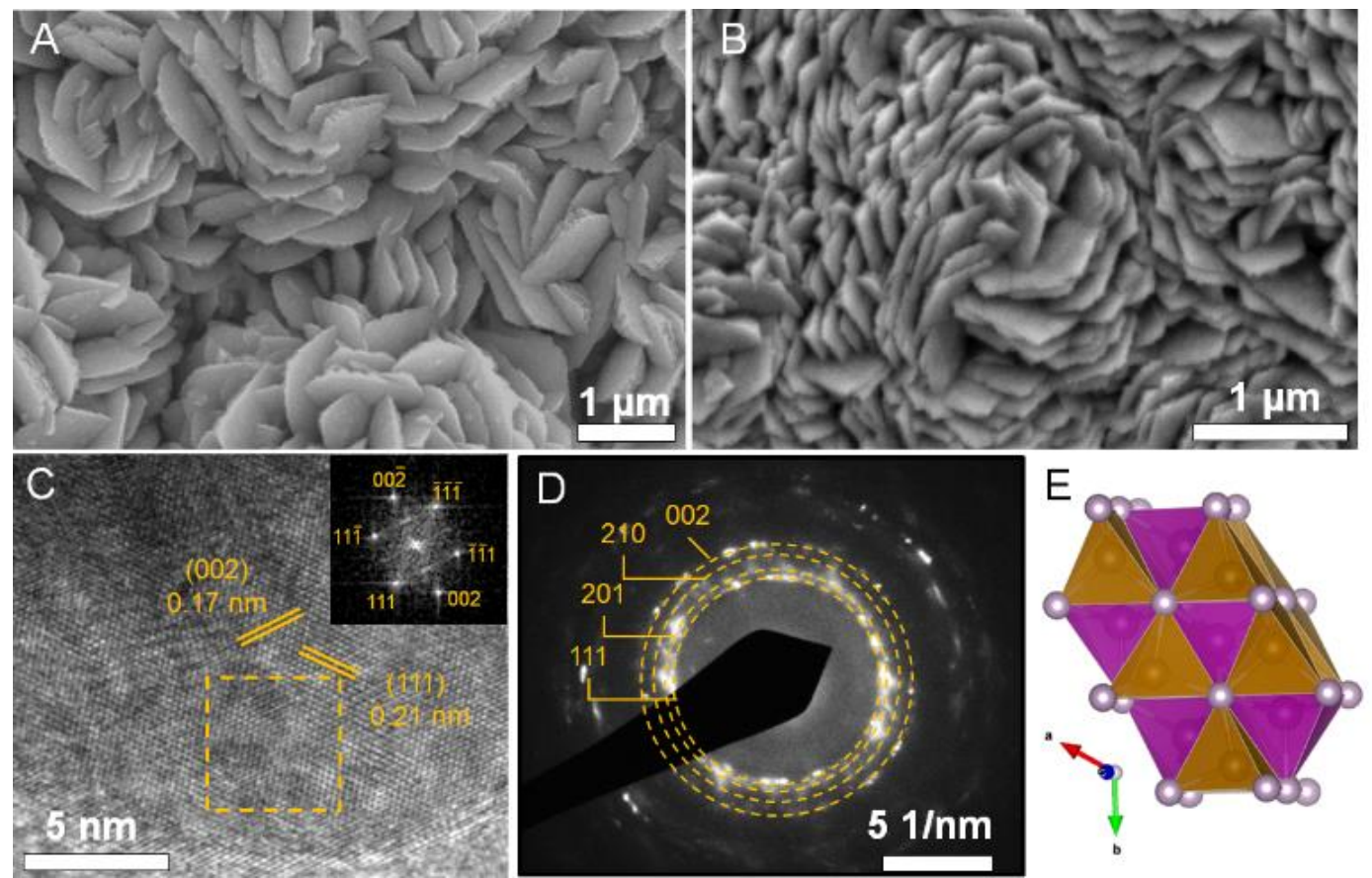

Fig. 1. Morphology characterization. SEM image of FeMnP on (A) NF and (B) GNF. (C) HRTEM of FeMnP. The inserted image is the Fast Fourier transform (FFT) image of the selected area in the dotted area. (D) SAED pattern of FeMnP. (E) Crystalline structure of hexagonal FeMnP. Grey spheres are P atoms; purple and yellow polyhedral are statistically mixed Fe and Mn atoms.

The high resolution transmission electron microscopy (HRTEM) image shows clear crystalline fringes with d-spacings of $0.17 \mathrm{~nm}$ and $0.21 \mathrm{~nm}$ (Fig. 1C), which are indexed to the (002) and (111) planes, in agreement with the FFT image, which displays a hexagonal spot pattern. Selected area electron diffraction (SAED) imaging shows a characteristic polycrystalline ring pattern 
(Fig. 1D). These rings can be indexed to the (111), (201), (210) and (002) planes, further confirming the hexagonal phase of FeMnP. Figure 1E shows the crystal structure of FeMnP in polyhedral view. FeMnP produced using FeMn $(\mathrm{CO})_{8}\left(\mu-\mathrm{PH}_{2}\right)$ has been shown to be in a hexagonal $P \overline{6} 2 m$ space group with the metals occupationally disordered over two sites [32].

The elemental composition was investigated by TEM energy dispersive spectroscopy (TEM-EDS) shown in Fig. 2A. The EDS spectrum (Fig. 2B) shows the $\mathrm{K}$ line signals of $\mathrm{Fe}, \mathrm{Mn}$ and $\mathrm{P}$. The image taken under secondary electron imaging (SEI) mode confirmed the uniform nanoplatelet structure of FeMnP (Fig. S6). The Fe, Mn and P atoms were homogeneously distributed across the whole FeMnP nanoplatelet. Using wavelength-dispersive X-ray spectroscopy (WDS), it was found that the atomic ratio of Fe:Mn:P was $1: 1.14: 1.05$, which is very close to the atomic ratio of $1: 1: 1$ of Fe:Mn:P in the precursor compound. Trace amounts of oxygen and $\mathrm{Ni}$ were also detected and originated from the surface oxide and nickel foam substrate, respectively. The chemical states of the as-deposited FeMnP were examined by sputtering assisted X-ray photoelectron spectroscopy (XPS) (Fig. 2C). Before sputtering, the strong peak in $\mathrm{Fe} 2 \mathrm{p}_{3 / 2}$ spectra at $706.9 \mathrm{eV}$ indicates that the surface $\mathrm{Fe}$ was metallic [41]. After 3 min and 6 min of sputtering, the Fe remained metallic. The surface $\mathrm{Mn}$ appears to be divalent given the peak at $641.9 \mathrm{eV}$ in its $2 \mathrm{p}_{3 / 2}$ spectrum [42]; broadening of its $\mathrm{Mn} 2 \mathrm{p}_{3 / 2}$ peak can be observed coupled to a shift of the binding energy toward $638.7 \mathrm{eV}$ after 3 min or 6 min of sputtering, 
implying the presence of metallic Mn. Surface P shows two broad peaks at $129.5 \mathrm{eV}$ and $133.4 \mathrm{eV}$ in its $2 \mathrm{p}$ spectrum, which are assigned to the phosphide and oxidized phosphorous components, [43] respectively. The latter is probably because of slight surface oxidation. After 3 min to 6 min of sputtering, the peak at $133.4 \mathrm{eV}$ disappeared and a doublet peak appeared at $129.4 \mathrm{eV}$ and $130.2 \mathrm{eV}$, which is indexed to phosphide $\mathrm{P}$. This suggests that all the $\mathrm{P}$ is in its phosphidic state below the surface. The atomic ratio of Fe: Mn: P from XPS analysis is $1: 1: 1$, consistent with the results of WDS measurements.

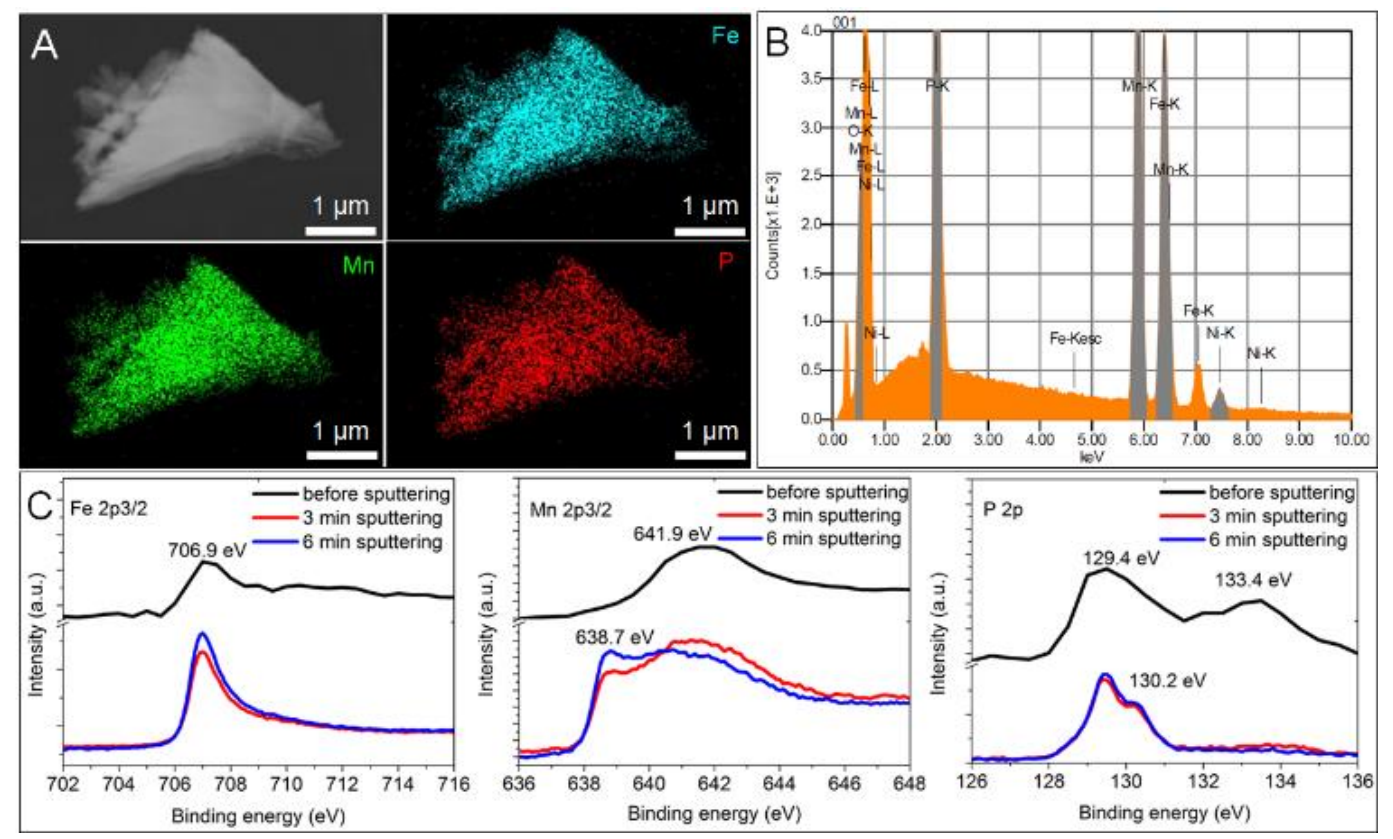

Fig. 2. Composition and chemical states of FeMnP. (A) TEM-EDS results of a single FeMnP platelet showing the existence and distribution of $\mathrm{Fe}, \mathrm{Mn}$ and $\mathrm{P}$. (B) composition analysis in EDS. (C) Chemical states of Fe, Mn and P by elementary XPS scanning of FeMnP before catalysis.

The FeMnP/NF and FeMnP/GNF prepared by the SSP-MOCVD method can be directly used as binder-free electrodes for water splitting. The loading amounts of 
FeMnP on NF and GNF were $2.6 \mathrm{mg} \mathrm{cm}^{-2}$ and $2.4 \mathrm{mg} \mathrm{cm}^{-2}$, respectively. The overpotential and Tafel slope are the two most important parameters for the evaluation of the catalytic activity of electrocatalyst [44, 45] Fig. 3A and B show OER polarization curves and their corresponding Tafel plots in a $0.1 \mathrm{M} \mathrm{KOH}$ aqueous solution. The overpotential is defined as the potential at which the current density reaches $10 \mathrm{~mA} \mathrm{~cm}^{-2}$ [3]. Both the bare NF and GNF electrodes show negligible OER performance in base. The FeMnP/NF electrode generated a current density of $10 \mathrm{~mA}$ $\mathrm{cm}^{-2}$ at a potential of $1.51 \mathrm{~V}$ versus the reversible hydrogen electrode (RHE) with a low overpotential of $280 \mathrm{mV}$ and a Tafel slope of $57 \mathrm{mV} \mathrm{dec}{ }^{-1}$. Thus, the OER overpotential and Tafel slope of FeMnP/NF are lower than most recently developed TMPs, including CoMnP nanoparticles (330 mV and $61 \mathrm{mV} \mathrm{dec}{ }^{-1}$ ) [20], NiCoP microspheres $\left(340 \mathrm{mV}\right.$ and $86 \mathrm{mV} \mathrm{dec}^{-1}$ ) [46], CoP nanorods $(290 \mathrm{mV}$ and $65 \mathrm{mV}$ $\left.\operatorname{dec}^{-1}\right)$ [29], and $\mathrm{Ni}_{2} \mathrm{P}\left(290 \mathrm{mV}\right.$ and $\left.59 \mathrm{mV} \mathrm{dec}^{-1}\right)$ [21], and other reported active OER electrocatalysts in Table S1. There is an observed enhancement compared to previous electrodes with FeMnP thin films on fluorine-doped tin oxide (FTO), which delivered an impressive overpotential of $300 \mathrm{mV}$ with a Tafel slope of $65 \mathrm{mV} \mathrm{dec}^{-1}$ despite the high resistivity of FTO [35]. Notably, nanoparticles of $\mathrm{Fe}_{1.1} \mathrm{Mn}_{0.9} \mathrm{P}$ were recently found to require an overpotential of $440 \mathrm{mV}$ for $10 \mathrm{~mA} \mathrm{~cm}$-2 which is markedly higher than the results presented here with a 1:1:1 ratio of Fe:Mn:P [47]. The difference in overpotentials between FeMnP/NF thin films and nanoparticulate $\mathrm{Fe}_{1.1} \mathrm{Mn}_{0.9} \mathrm{P}$ can largely be ascribed to high quality ohmic contact of FeMnP/NF and the lack of an oxide layer between the FeMnP and the NF. By depositing FeMnP on 


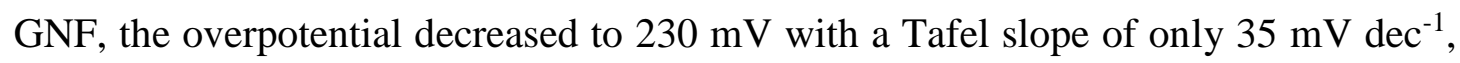
making the FeMnP/GNF among the best OER catalysts (Table S1). The calculated turnover frequency (TOF) in OER for FeMnP/GNF at an overpotential of $280 \mathrm{mV}$ was $0.28 \mathrm{~s}^{-1}$, suggesting an intrinsically high OER catalytic activity of FeMnP.
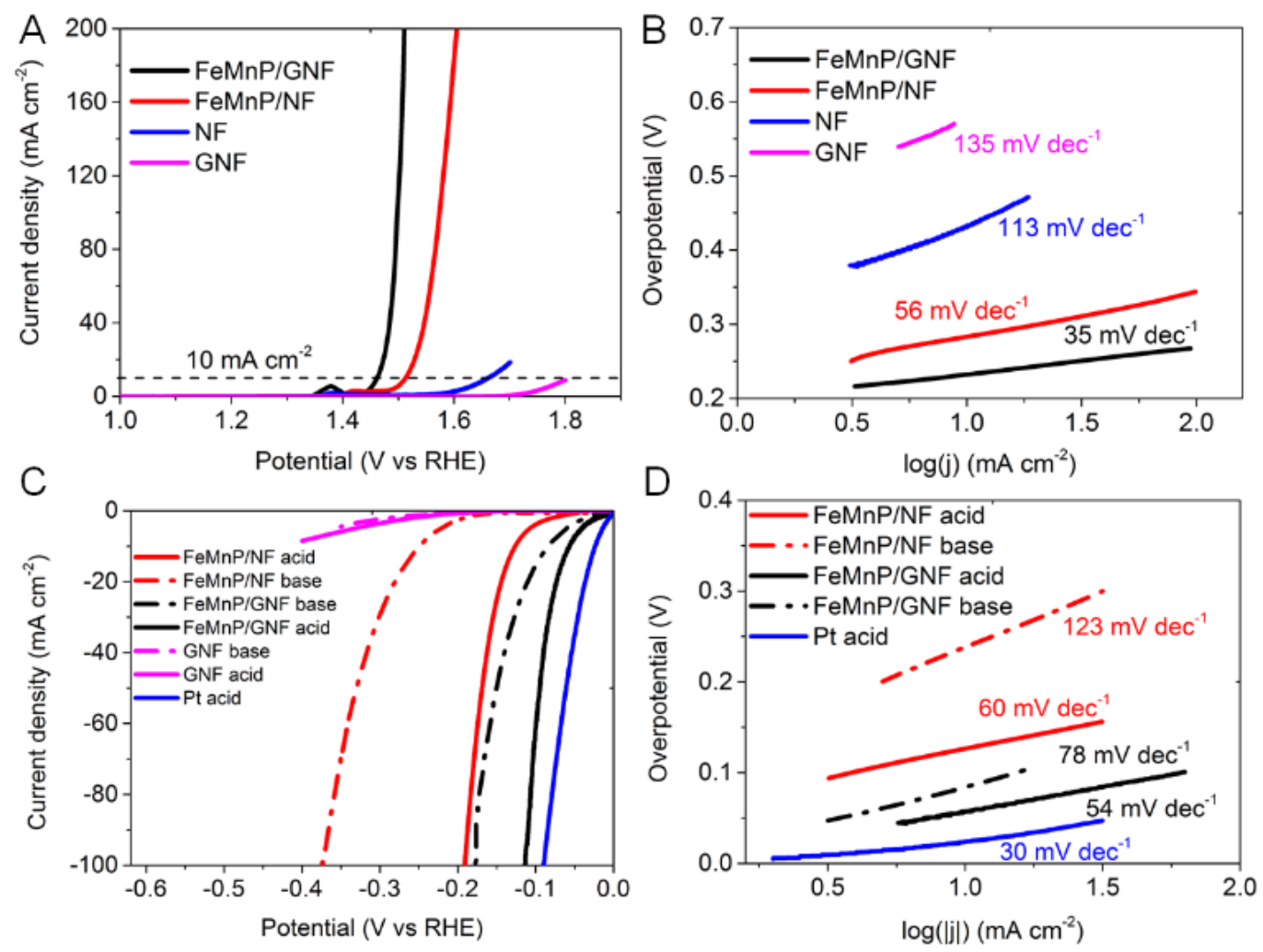

Fig. 3. Electrocatalytic activity characterization. (A) The OER polarization curves in $0.1 \mathrm{M} \mathrm{KOH}$ at scan rate of $5 \mathrm{mV} \mathrm{s}^{-1}$. (B) The OER Tafel plots. (C) The HER polarization curves in $0.5 \mathrm{M} \mathrm{H}_{2} \mathrm{SO}_{4}$ and $0.1 \mathrm{M} \mathrm{KOH}$ at scan rate of $5 \mathrm{mV} \mathrm{s}^{-1}$. (D) The corresponding HER Tafel plots.

Besides the outstanding OER activity, the FeMnP/NF and FeMnP/GNF electrodes are also highly active towards HER in acid. Fig. 3C and D show the polarization curves and corresponding Tafel slopes in $0.5 \mathrm{M} \mathrm{H}_{2} \mathrm{SO}_{4}$. The FeMnP/NF electrode shows high 
HER activity with an overpotential of $125 \mathrm{mV}$ and a Tafel slope of $60 \mathrm{mV} \mathrm{dec}{ }^{-1}$. The HER performance of FeMnP is better than, or comparable to, other earth- abundant HER catalysts, including the transition metal sulfides $\mathrm{MoS}_{2}[48,49]$, the TMPs FeP [50], $\mathrm{Ni}_{2} \mathrm{P}$ [51] and WP [52]. An even lower overpotential toward HER was achieved by depositing FeMnP on GNF. The FeMnP/GNF electrode required an overpotential of as low as $57 \mathrm{mV}$ with the Tafel slope of $54 \mathrm{mV} \mathrm{dec}{ }^{-1}$, which matches the performance characteristics of other highly active HER catalysts listed in Table S2. Notably, the exchange current density increased from $0.14 \mathrm{~mA} \mathrm{~cm}{ }^{-2}$ for FeMnP/NF to $1.0 \mathrm{~mA} \mathrm{~cm}^{-2}$ when FeMnP/GNF is used, and is only marginally lower than the highest recorded number of $1.2 \mathrm{~mA} \mathrm{~cm} \mathrm{~cm}^{-2}$ by graphitic carbon supported nickel [53]. The calculated TOF for the HER at an overpotential of $100 \mathrm{mV}$ for FeMnP/GNF was 0.14 $\mathrm{s}^{-1}$.The HER activity in base was tested with the objective being total water splitting from one type of electrode in the same cell. FeMnP shows high HER activity in $0.1 \mathrm{M}$ $\mathrm{KOH}$. The HER overpotentials of the FeMnP/NF and FeMnP/GNF electrodes in base were $249 \mathrm{mV}$ and $84 \mathrm{mV}$, respectively, with corresponding Tafel slopes of $123 \mathrm{mV}$ $\operatorname{dec}^{-1}$ and $78 \mathrm{mV} \operatorname{dec}^{-1}$. The exchange current densities for FeMnP/NF and FeMnP/GNF in base were $0.14 \mathrm{~mA} \mathrm{~cm}^{-2}$ and $0.78 \mathrm{~mA} \mathrm{~cm}^{-2}$. 

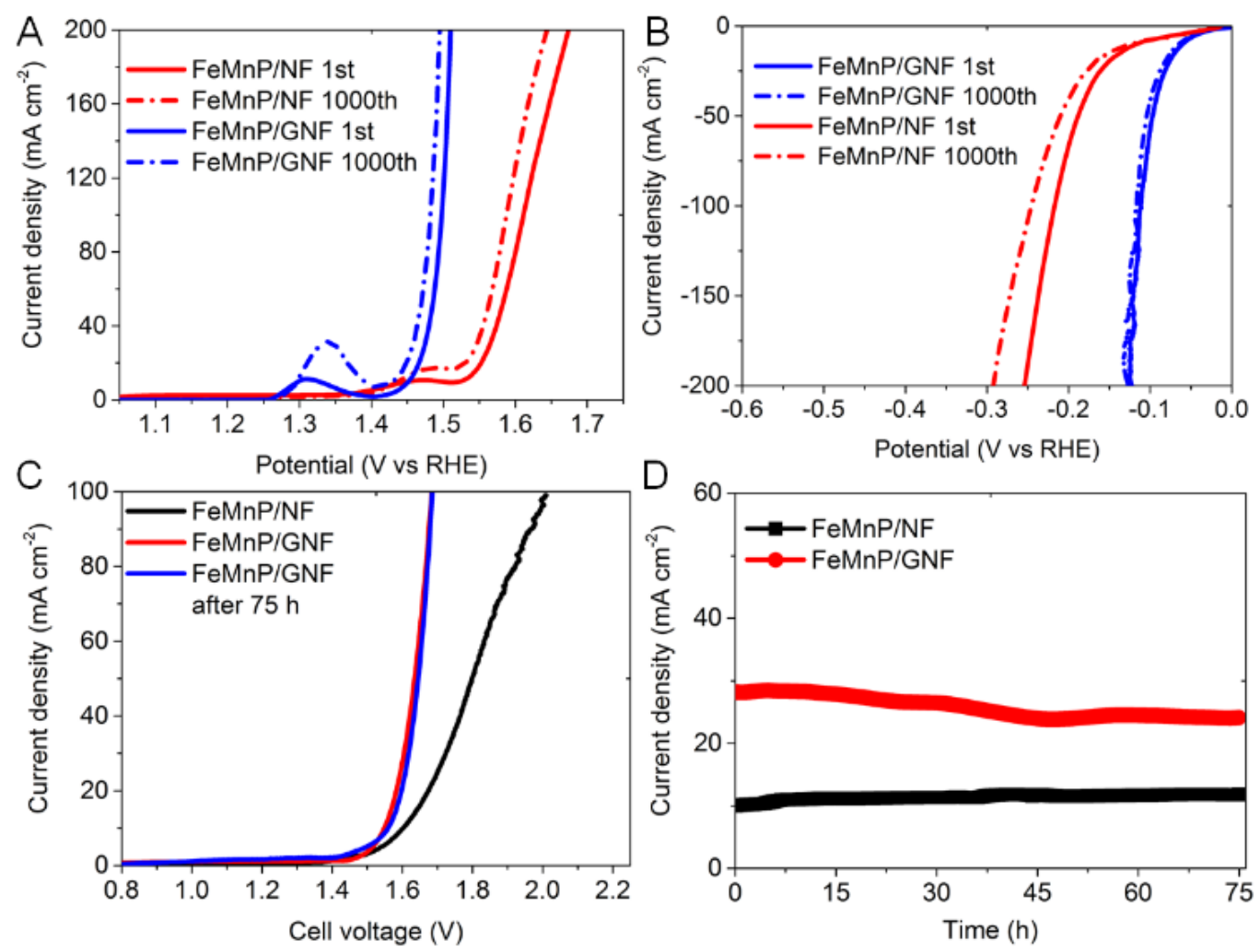

Fig. 4. Stability characterization and overall water splitting. (A) OER polarization curves of the FeMnP/NF and FeMnP/GNF electrodes in $0.1 \mathrm{M} \mathrm{KOH}$ at scan rate of $100 \mathrm{mV} \mathrm{s}^{-1}$, showing the $1^{\text {st }}$ cycle and the $1000^{\text {th }}$ cycle. (B) HER polarization curves of the FeMnP/NF and FeMnP/GNF electrodes in $0.5 \mathrm{M} \mathrm{H}_{2} \mathrm{SO}_{4}$ at scan rate of $100 \mathrm{mV}$ $\mathrm{s}^{-1}$, showing the $1^{\text {st }}$ cycle and the $1000^{\text {th }}$ cycle. (C) $I-V$ curves of the two electrode water splitting using FeMnP as bifunctional catalyst in $0.1 \mathrm{M} \mathrm{KOH}$ at scan rate of 5 $\mathrm{mV} \mathrm{s}^{-1}$. (D) Long-term stability at a constant cell voltage of $1.6 \mathrm{~V}$ for 75 hours using two FeMnP/NF electrodes or two FeMnP/GNF electrodes.

To assess the electrode for practical use, the OER stability was evaluated by cycling the FeMnP/NF and FeMnP/GNF electrodes in $0.1 \mathrm{M} \mathrm{KOH}$ for 1000 cycles at $100 \mathrm{mV}$ $\mathrm{s}^{-1}$, as shown in Fig. 4A. It is noted that there is an obvious oxidation peak between 
1.3 $\mathrm{V}$ and $1.4 \mathrm{~V}$ for FeMnP/GNF, which can be assigned to the Ni oxidation [54]. This phenomena is common in many OER electrodes using Nickel foam the substrate $[54,55]$. Upon cycling, the overpotential for the FeMnP/NF electrode to reach an anodic current density of $20 \mathrm{~mA} \mathrm{~cm}{ }^{-2}$ decreased from the initial $320 \mathrm{mV}$ ( $1^{\text {st }}$ cycle) to $300 \mathrm{mV}$ (the $1000^{\text {th }}$ cycle), indicating an improvement of the OER catalytic activity. A similar increase was also observed for the FeMnP/GNF electrode with the overpotential at $20 \mathrm{~mA} \mathrm{~cm}^{-2}$ decreasing from $240 \mathrm{mV}$ to $220 \mathrm{mV}$. The HER stability was conducted by cycling the FeMnP/NF and FeMnP/GNF electrodes in $0.5 \mathrm{M}$ $\mathrm{H}_{2} \mathrm{SO}_{4}$ over 1000 cycles at $100 \mathrm{mV} \mathrm{s}^{-1}$ (Fig. 4B). For the FeMnP/NF electrode, the HER performance shows a slight decay with the overpotential at $20 \mathrm{~mA} \mathrm{~cm}^{-2}$ increasing from the initial $150 \mathrm{mV}$ to $168 \mathrm{mV}$. However, for the FeMnP/GNF electrode, the profile of the polarization curve at the $1^{\text {st }}$ cycle was the same as that at the $1000^{\text {th }}$ cycle, indicating optimal HER stability.

Our results for the HER and OER half reactions suggest that FeMnP could be an active and stable electrocatalyst for overall water splitting. We assessed its overall water splitting activity in $0.1 \mathrm{M} \mathrm{KOH}$ solution by pairing two FeMnP/NF electrodes or two FeMnP/GNF electrodes. The cell voltage for FeMnP/NF was $1.60 \mathrm{~V}$ to reach $10 \mathrm{~mA} \mathrm{~cm}^{-2}$ current density, while a lower cell voltage of $1.55 \mathrm{~V}$ at $10 \mathrm{~mA} \mathrm{~cm}{ }^{-2}$ was obtained by using two FeMnP/GNF electrodes (Fig. 4C). The cell voltage of FeMnP/GNF is better than or comparable to previously reported bifunctional electrocatalysts, such as $\mathrm{NiCo}_{2} \mathrm{~S}_{4}(1.63 \mathrm{~V})$ [56], $\mathrm{Ni}_{2} \mathrm{P}$ (1.63 V) [21], $\mathrm{NiCoP}(1.64 \mathrm{~V})$ [46], $\mathrm{NiCo}_{2} \mathrm{O}_{4}(1.65 \mathrm{~V})$ [57], and those bifunctional electrocatalysts in Table S3. Both 
the FeMnP/NF and FeMnP/GNF electrodes show extraordinary long-term stability for overall water splitting in base. The current density of the FeMnP/NF electrode at 1.60 V shows no decay (Fig. 4D). For the FeMnP/GNF electrode, at the same cell voltage of $1.60 \mathrm{~V}$ after 75 hours of testing, the current density slightly decreased from initial $27.5 \mathrm{~mA} \mathrm{~cm}^{-2}$ to $25 \mathrm{~mA} \mathrm{~cm}^{-2}$. The polarization curve over 75 hours testing was almost identical to the initially obtained curve (Fig. 4C). Fig. S7 shows that the produced $\mathrm{O}_{2}$ and $\mathrm{H}_{2}$ amounts measured by gas chromatography (GC) match the theoretically calculated amounts of $\mathrm{O}_{2}$ and $\mathrm{H}_{2}$ during the overall water splitting over two FeMnP/NF or two FeMnP/GNF electrodes. The molar ratio of $\mathrm{H}_{2}$ to $\mathrm{O}_{2}$ is close to 2, suggesting almost $100 \%$ of the Faradaic efficiency for both FeMnP/NF and FeMnP/GNF.

A good electrocatalyst with high activity is defined by high electrical conductivity and a large electrochemically active surface area (ECSA). The Nyquist plots for the electrodes in Figure S8 show that the ohmic resistance decreased after nickel foam was coated by CVD grown multilayer graphene coupled with a decrease in the charge transfer resistance for both the OER and HER. Graphene has high electrical conductivity and surface area, is very stable in acid and strong base, and can also protect the surface of the nickel foam from oxidation. It is suggested that graphene with its superior electron pathway provides a strongly coupled interface between the active phase and current collector.[58, 59] We further compared the ECSA of FeMnP/NF and FeMnP/GNF, which was estimated from the double-layer capacitance $\left(\mathrm{C}_{\mathrm{dl}}\right)$ (Figure S9). The FeMnP/GNF is calculated to have an ECSA of $71 \mathrm{mF} \mathrm{cm} \mathrm{cm}^{-2}$, 
about $20 \%$ higher than the ECSA of $57 \mathrm{mF} \mathrm{cm}^{-2}$ of FeMnP/NF, and both of them are much higher than that of bare NF of $1.4 \mathrm{mF} \mathrm{cm}^{-2}$. The greater ECSA after FeMnP deposition on NF contributes to the high activity of FeMnP.

We conducted a chronoamperometry measurement of the FeMnP/NF at an overpotential of $300 \mathrm{mV}$ in $0.1 \mathrm{M} \mathrm{KOH}$ for OER (Fig. S10) that showed a stable current density over 20 hours, after which the OER electrode was analysed by WDS and sputtering assisted XPS. The images of the OER electrode taken under SEI mode show the phase homogeneity of the tested FeMnP which retained the nanoplatelet morphology (Fig. S11), while the images taken under backscattered electron emission (BSE) mode show the phase uniformity of the tested FeMnP. Further composition analysis by WDS confirmed the presence of $\mathrm{Fe}, \mathrm{Mn}$ and $\mathrm{P}$ with an atomic ratio of 1:1.14:0.9 (Fig. S12), which is similar to the values found before catalysis $(1: 1.14$ : 1.05). XPS analysis (Fig. S13) of the OER tested electrode indicated slight surface oxidation given the binding energies of surface Fe and Mn were $710.9 \mathrm{eV}$ and 641.9 $\mathrm{eV}$, corresponding to the $\mathrm{FeO}_{\mathrm{x}}[60]$ and $\mathrm{MnO}_{\mathrm{x}}$ [42]. Furthermore, $\mathrm{P}$ was not detected at the surface. While the lack of phosphorus appears to disagree with the results of the WDS analysis, the difference can be accounted for by comparing the two techniques. WDS samples a much wider and deeper cross-section of sample $\left(1 \mu \mathrm{m}^{3}\right)$ whereas XPS is limited to the upper $10 \mathrm{~nm}$. Thus, FeMnP persists under the oxidized surface. This is consistent with the result of our previous work [35]. The binding energy at 530.0 $\mathrm{eV}$ in $\mathrm{O} 1 \mathrm{~s}$ spectra also confirmed the existence of $\mathrm{FeO}_{\mathrm{x}}$ and $\mathrm{MnO}_{\mathrm{x}}[42,60]$. We then used sputtering to remove the surface species and conducted XPS analysis. Fe, Mn 
and $\mathrm{P}$ were still in their zero-valence states below the surface. Therefore, it is concluded that over the course of the OER testing, the surface of FeMnP was oxidized to form active sites with the pristine underlying metallic FeMnP layer providing a highly conductive electron pathway, a configuration responsible for the high stability and performance of the FeMnP catalyst. Sputter assisted XPS analysis was also used to analyze the FeMnP/NF electrode after a long-term HER stability test in acid (Fig. S10 and S14).

To elucidate the underlying fundamental properties of FeMnP for high HER activity, we performed a series of DFT calculations as summarized in Fig. 5. The computational methods are detailed in the Supplementary Information. Predictions from the Bravais-Friedel-Donnay-Harker (BFDH) algorithm indicate that (100) and (001) low index facets are the most probable terminations for FeMnP [61]. A calculation of the surface formation energy of a FeMnP (100) resulted in $0.11 \mathrm{eV} / \AA^{2}$, while the cleavage of FeMnP along (001) planes requires $0.42 \mathrm{eV} / \AA^{2}$ to yield $\mathrm{Fe}$ - and Mn- terminated surfaces. The low energy required to form (100) facets is a good indication of dominant FeMnP (100) surfaces that may serve as active facets for electrocatalytic HER. Of the two different terminations exhibited by the less stable (001) facet, we concentrate here on the Mn-terminated surface, referred to as (001)-Mn. The Fe-terminated FeMnP (001) facet strongly exhibits strongly endergonic binding after the preferred adsorption sites are occupied [4]. 


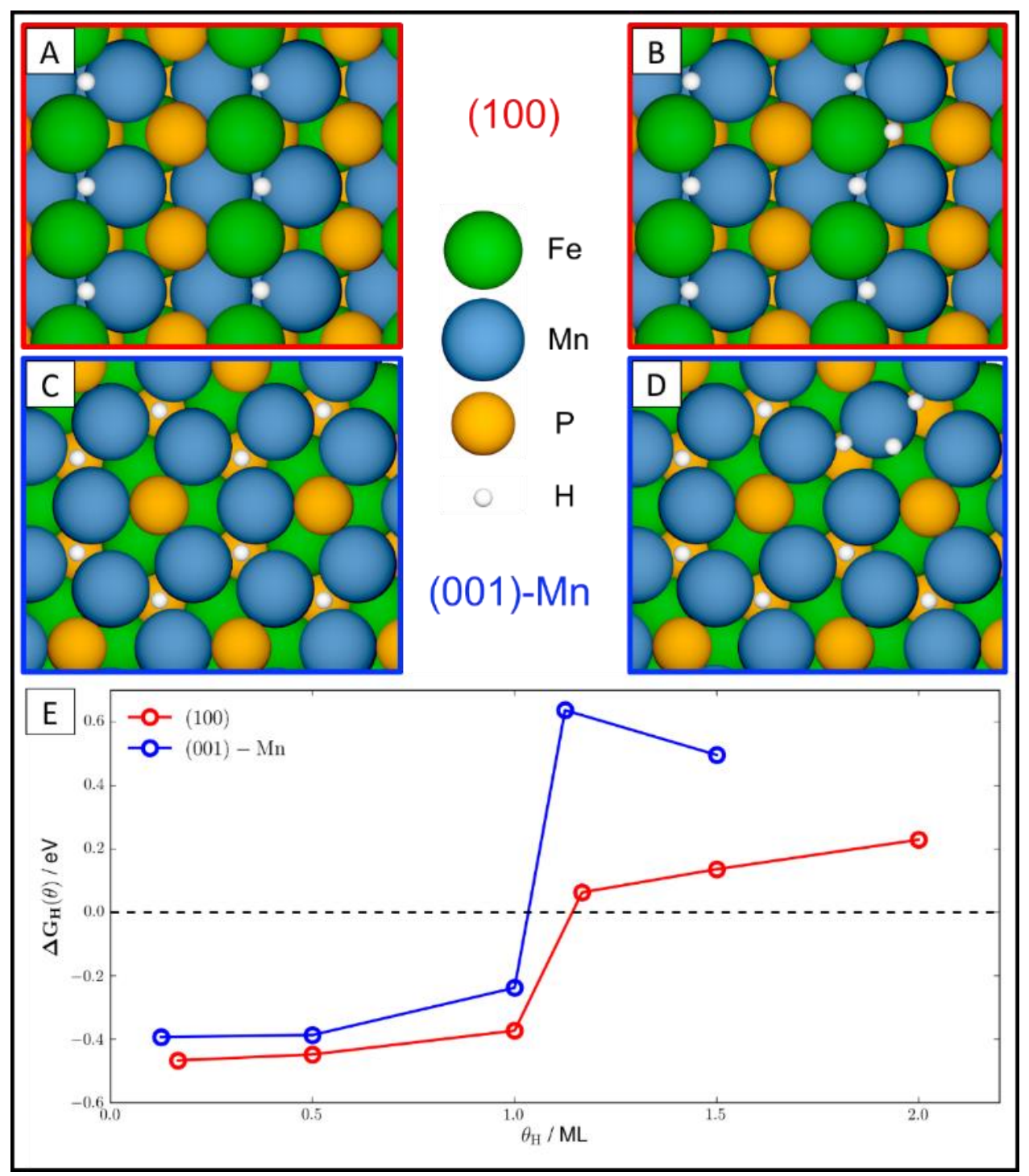

Fig. 5. Coverage-dependent hydrogen binding on (100) and (001)-Mn facets of FeMnP. Panels (A) and (B) show hydrogen adsorbed on FeMnP (100) at $1 \mathrm{ML}$ and 7/6 ML, respectively. Panels (C) and (D) depict hydrogen adsorbed on FeMnP (001)-Mn surface at $1 \mathrm{ML}$ and 9/8 ML, respectively. Panel (E) shows the calculated differential free binding energy of hydrogen $\Delta G_{H}$ as a function of coverage. ML: monolayer.

A necessary, but not sufficient, criterion for optimal HER performance is a differential Gibbs free binding energy for hydrogen, $\Delta G_{H}$, close to zero. This 
criterion can be rationalized in terms of the Sabatier principle and the competing hydrogen binding requirements for facile proton adsorption via the Volmer step and rapid $\mathrm{H}_{2}$ evolution by either the Tafel or Heyrovsky reaction [62]. Fig. 5E shows $\Delta G_{H}$ as a function of coverage $\theta$ on the (100) and (001)-Mn facets. The preferred hydrogen adsorption site is between a Fe-Fe bridge site and a Mn atom on the (100) facet depicted in Fig. 5A, and threefold Mn sites on the (001) facet (Fig. 5C). With increasing hydrogen coverage up to $1 \mathrm{ML}$ hydrogen atoms continue to occupy their preferred adsorption sites, and small repulsive interactions contribute to gradually weaker binding. Above $1 \mathrm{ML}$ the differential $\Delta G_{H}$ on both surfaces changes from exergonic to endergonic, which corresponds to the expected surface coverage under electrocatalytic operating conditions.

For the (001)-Mn facet (blue) at 1 ML coverage we obtain the smallest magnitude of $\Delta G_{H}=-0.24 \mathrm{eV}$, which indicates overbinding. Under these conditions, a subsequent hydrogen adsorption at 9/8 ML is highly unstable and requires $+0.63 \mathrm{eV}$. This behaviour is reminiscent of pure $\mathrm{Fe}_{2} \mathrm{P}$ and corresponds to the occupation of an unfavourable binding site near a Mn atom, which also causes a small displacement of two $\mathrm{H}$ atoms in the preferred threefold Mn site (Fig. 5D). In contrast, hydrogen binding to the (100) facet (red) is almost thermoneutral with $\Delta G_{H}=0.06 \mathrm{eV}$ at $7 / 6$ ML coverage. In this case, hydrogen adsorbs between a Mn-Mn bridge site and an Fe atom, while forcing two other hydrogen atoms onto Fe-Fe bridge positions (Fig. 5B). Furthermore, $\Delta G_{H}$ of the (100) facet shows a weak coverage-dependence and remains less than $0.23 \mathrm{eV}$ up to $2 \mathrm{ML}$. This behaviour suggests a good tolerance to 
coverage variations and the possibility of favourable entropy contributions stemming from a large number of surface configurations that hydrogen atoms can assume in the shallow binding energy potential. Overall, the hydrogen binding characteristics of the FeMnP (100) surface fulfill the necessary requirement for efficient hydrogen evolution at its optimal coverage and further suggest that the favourable characteristics extend to even higher coverage. We note that the relevant hydrogen binding sites on FeMnP (100) are comprised of mixed Fe and Mn atoms, which points to a synergistic effect between these constituents and is fully consistent with the electrocatalytic characterization results in this work.

\section{Conclusions}

FeMnP was grown on NF and GNF by a facile MOCVD method in which the stoichiometry was controlled by use of an atomically precise single-source precursor. FeMnP was proven to be an efficient bifunctional catalyst showing remarkable activity towards the HER and the OER individually and in tandem with robust stability. Protecting NF with conductive multilayer-graphene prior to depositing FeMnP resulted in further performance improvements. The ability of the MOCVD method to grow uniform films of a phase pure, active catalyst on 3-dimensional structures, such as demonstrated here with NF/GNF, suggests that it is a practical route to preparing electrode materials. We believe that this study will facilitate the development of new bifunctional catalysts based on transition metal phosphides. 


\section{Experimental Section}

\subsection{Growth of Graphene}

Nickel foam was used as the substrate for the chemical vapor deposition (CVD) growth of graphene according to reported literature methods [63]. Before graphene growth, the nickel foam was cut into pieces of $10 \mathrm{~mm} \times 30 \mathrm{~mm}$, and ultrasonicated for 5 minutes sequentially in deionized water, diluted $\mathrm{HCl}$ solution, ethanol, and acetone. The clean nickel foam was placed in the center of a quartz boat which was placed in the center of a quartz tube. The quartz tube was then evacuated and filled with Ar gas. The vacuum-filling process was repeated twice. The nickel foam was heated to $1000{ }^{\circ} \mathrm{C}$ under $\mathrm{Ar}$ gas (600 s.c.c.m). $\mathrm{H}_{2}$ (200 s.c.c.m) was then introduced into the tube to clean and eliminate the oxidized surface of nickel foam. After $30 \mathrm{~min}$ of hydrogen flow, $\mathrm{CH}_{4}$ (3 s.c.c.m) was introduced for the growth of graphene. After 5 min of reaction, the feeding of $\mathrm{CH}_{4}$ stopped and the sample was rapidly moved out from the heating zone and cooled to room temperature under $\mathrm{Ar}\left(600\right.$ s.c.c.m) and $\mathrm{H}_{2}$ (200 s.c.c.m).

\subsection{Deposition of FeMnP}

FeMnP was deposited onto the surface of bare nickel foam and graphene protected nickel foam. Before the deposition, the nickel foam was also cleaned with the same procedure used in the growth of graphene. The organometallic precursor $\mathrm{FeMn}(\mathrm{CO})_{8}\left(\mu-\mathrm{PH}_{2}\right)$ was synthesized according to a previous report [36], and used as the precursor for the deposition of FeMnP with a home-built metal-organic chemical 
vapor deposition (MOCVD) apparatus (Fig. S1) [31]. The substrates (nickel foam or graphene-protected nickel foam) were oriented vertically and affixed to end of a stainless steel heating stage (Fig. S1) with silver paste and heated at $130{ }^{\circ} \mathrm{C}$ in air to cure the silver paste and provide good thermal contact. $20 \mathrm{mg}$ of the precursor $\mathrm{FeMn}(\mathrm{CO})_{8}\left(\mu-\mathrm{PH}_{2}\right)$ was loaded into the end of the tube apparatus in a glove box under a nitrogen atmosphere. The substrate-affixed heating stage was placed in the apparatus and sealed. Then it was transferred to a high-vacuum manifold and the end of the apparatus with the precursor was submerged in a liquid nitrogen bath. While the precursor was kept at $77 \mathrm{~K}$, the apparatus was evacuated until a cold-cathode ionization vacuum gauge stabilized at approximately $2.0 \times 10^{-6}$ Torr. The position of the heating stage in the apparatus was adjusted to achieve optimum substrate coverage. Zone 1 of the apparatus, where the substrates were located, was then preheated to 350 ${ }^{\circ} \mathrm{C}$ for 30 minutes using a heating tape. The nitrogen bath was removed from the precursor which was allowed to warm to room temperature. After 20 minutes, a metallic film had formed on the substrates and walls surrounding the substrate assembly. The apparatus was disassembled under a nitrogen atmosphere and then the material was stored in air.

\subsection{Characterization}

The structure of the samples were characterized by SEM with an FEI Quanta 400 FEG microscope operated at $5 \mathrm{kV}$ and a TEM (JEOL 2010H) operated at $200 \mathrm{kV}$. The element composition was analyzed by EDS mapping and wavelength dependent 
dispersive X-ray spectroscopy (WDS) with a JEOL JXA 8530F Hyperprobe. X-ray photoelectron spectroscopy (XPS) was carried out on a Physical Electronics PHI Quantera SXM instrument using a monochromatic Al $\mathrm{K} \alpha$ source (1486.6 eV) operated at $40.7 \mathrm{~W}$ with a beam size of $200 \mu \mathrm{m}$ and a take-off angle of $45^{\circ}$. Sputtering was performed with by $2 \mathrm{~mm} 3 \mathrm{keV} \mathrm{Ar}{ }^{+}$ion beam with $0.5 \mathrm{~mA}$ current. The X-ray powder diffraction (XRD) was conducted on a SIEMENS Diffractometer D5000 equipped with a $\mathrm{Cu}$ target. The Raman spectra were recorded on a Horiba iHR320 spectrometer with an excitation wavelength of $532 \mathrm{~nm}$. Catalyst loading amounts were determined by taking the difference in weight before and after FeMnP deposition.

\subsection{Electrochemical measurements}

All electrochemical tests were performed with a 263A Princeton Applied Research (PAR) potentialstat/galvanostat Instrument. Linear sweep voltammetry (LSV) was performed at a scan rate of $5 \mathrm{mV} \mathrm{s}^{-1}$, and cyclic voltammetry (CV) was performed at a scan rate of $100 \mathrm{mV} \mathrm{s}^{-1}$ for the stability test. Electrochemical impedance spectroscopy (EIS) was performed at an $\mathrm{AC}$ amplitude of $10 \mathrm{mV}$ in a frequency range of 100000 $\mathrm{Hz}$ to $10 \mathrm{mHz}$ at an overpotential of $200 \mathrm{mV}$ for the HER and $300 \mathrm{mV}$ for the OER. Measurements were conducted in a three-electrode setup using $\mathrm{Ag} \mid \mathrm{AgCl}$ and a $\mathrm{Pt}$ plate $\left(1 \times 1 \mathrm{~cm}^{2}\right)$ as the reference electrode and counter electrode, respectively. A 0.5 $\mathrm{M} \mathrm{H}_{2} \mathrm{SO}_{4}$ aqueous solution and $0.1 \mathrm{M} \mathrm{KOH}$ aqueous solution were used as the electrolyte for HER and OER, respectively. IR correction was automatically made by 
the software equipped with the PAR instrument at $85 \%$ compensation. Both the electrolytes were degassed with $\mathrm{N}_{2}$ for 30 min before use and continuously bubbled during the tests. The FeMnP deposited nickel foam (FeMnP/NF) and graphene protected nickel foam (FeMnP/GNF) were directly used as the working electrode. Before use, samples were cut into the fixed size and then wired using copper wire with silver paste. Epoxy was used to cover the silver paste and the sample with exposed area of $0.2 \mathrm{~cm}^{-2}$. The potential versus that of reversible hydrogen electrode (RHE) was calculated with reference to $\mathrm{Ag} \mid \mathrm{AgCl}$ according to the Nernst equation: $\mathrm{E}_{\mathrm{RHE}}=\mathrm{E}_{\mathrm{Ag} \mid \mathrm{AgCl}}+0.05916 \times \mathrm{pH}+\mathrm{E}_{\mathrm{o}}$, where $\mathrm{E}_{\mathrm{RHE}}$ is the potential vs RHE, $\mathrm{E}_{\mathrm{Ag} \mid \mathrm{AgCl}}$ is the measured potential vs $\mathrm{Ag} \mid \mathrm{AgCl}$, and $\mathrm{E}_{\mathrm{o}}=0.2 \mathrm{~V}$ at $25^{\circ} \mathrm{C}$. The $\mathrm{pH}$ values of $0.5 \mathrm{M}$ $\mathrm{H}_{2} \mathrm{SO}_{4}$ and $0.1 \mathrm{M} \mathrm{KOH}$ were 0.3 and 13.0 at $25{ }^{\circ} \mathrm{C}$, respectively. The scan rate for the measurement of electrochemically active surface area (ECSA) was $10 \mathrm{mV} \mathrm{s}^{-1}, 20 \mathrm{mV}$ $\mathrm{s}^{-1}, 30 \mathrm{mV} \mathrm{s}^{-1}, 40 \mathrm{mV} \mathrm{s}^{-1}, 50 \mathrm{mV} \mathrm{s}^{-1}, 60 \mathrm{mV} \mathrm{s}^{-1}, 70 \mathrm{mV} \mathrm{s}^{-1}$, and $80 \mathrm{mV} \mathrm{s}^{-1}$ in the non-Faradaic reaction potential range. The overall water splitting was conducted by pairing two FeMnP/NF electrodes or two FeMnP/GNF electrodes in $0.1 \mathrm{M} \mathrm{KOH}$ electrolyte. The LSV was collected at the scan rate of $5 \mathrm{mV} \mathrm{s}^{-1}$. The chronoamperometry measurement for the long-term stability test was conducted at overpotential of $370 \mathrm{mV}$. The gaseous products of the overall water splitting for FeMnP/NF or FeMnP/GNF were analyzed using a gas chromatograph (GC) equipped with a thermal conduction detector (TCD) with $\mathrm{Ar}$ as the carrier gas, which was calibrated with highly pure $\mathrm{H}_{2}$ and $\mathrm{O}_{2}$ in advance, respectively. The overall water splitting was conducted at the cell voltage of $1.6 \mathrm{~V}$ in $0.1 \mathrm{M} \mathrm{KOH}$ which was 
degassed for $1 \mathrm{~h}$ in advance. The Faradaic efficiency was determined by comparing the amount of experimentally produced $\mathrm{H}_{2}$ and $\mathrm{O}_{2}$ during water electrolysis to the amount of theoretically calculated $\mathrm{H}_{2}$ and $\mathrm{O}_{2}$.

Acknowledgements

The authors thank Rice University, the National Science Foundation (CHE-1411495), and the Robert A. Welch Foundation (C-0976) for funding. This material is based upon work supported by the National Science Foundation Graduate Research Fellowship (DES) under Grant No. 1450681. The authors thank Dr. Gelu Costin for help with WDS measurements. Jiming Bao acknowledges support from the Robert A. Welch Foundation (E-1728). Zhenhuan Zhao thanks support from National Science Foundation for Post-doctoral Scientists of China (2015M582538). Zhiming Wang thanks financial support from 111 project (No. B13042). Hari Thirumalai and Lars C. Grabow acknowledge financial support from NSF-CBET 1605331 and NSF CAREER award number 1454384, respectively. Computational resources were provided by the uHPC cluster managed by the University of Houston and acquired through NSF-MRI award number 1531814. This work used the Extreme Science and Engineering Discovery Environment (XSEDE), which is supported by National Science Foundation grant number ACI-1053575. We also acknowledge the use of computational resources provided by the National Energy Research Scientific Computing (NERSC) Center, a DOE Office of Science User Facility supported by the Office of Science of the U. S. Department of Energy under contract number DE-AC02-05CH11231. Finally, we thank the Center of Advanced Computing and 
Data Systems (CACDS) at the University of Houston for access to the Maxwell/Opuntia HPC clusters.

\section{References}

[1] N. S. Lewis, Science 351 (2016) aad1920.

[2] Y. Jiao, Y. Zheng, M. Jaroniec and S. Z. Qiao, Chem. Soc. Rev. 44 (2015) 2060-2086.

[3] C. C. McCrory, S. Jung, J. C. Peters and T. F. Jaramillo, J. Am. Chem. Soc. 135 (2013) 16977-16987.

[4] J. Kibsgaard, C. Tsai, K. Chan, J. D. Benck, J. K. Nørskov, F. Abild-Pedersen and T. F. Jaramillo, Energy Environ. Sci. 8 (2015) 3022-3029.

[5] Y. Shi and B. Zhang, Chem. Soc. Rev. 45 (2016) 1529-1541.

[6] J. F. Callejas, C. G. Read, C. W. Roske, N. S. Lewis and R. E. Schaak, Chem. Mater. 28 (2016) 6017-6044.

[7] F. Wang, Y. Sun, Y. He, L. Liu, J. Xu, X. Zhao, G. Yin, L. Zhang, S. Li, Q. Mao, Y. Huang, T. Zhang and B. Liu, Nano Energy 37 (2017) 1-6.

[8] M. Zeng, Y. Chen, J. Li, H. Xue, R. G. Mendes, J. Liu, T. Zhang, M. H. Rümmeli and L. Fu, Nano Energy 33 (2017) 356-362.

[9] J.-S. Li, Y. Wang, C.-H. Liu, S.-L. Li, Y.-G. Wang, L.-Z. Dong, Z.-H. Dai, Y.-F. Li and Y.-Q. Lan, Nat. Commun. 7 (2016).

[10] X. Lü, A. Chen, Y. Luo, P. Lu, Y. Dai, E. Enriquez, P. Dowden, H. Xu, P. G. Kotula, A. K. Azad, D. A. Yarotski, R. P. Prasankumar, A. J. Taylor, J. D. Thompson and Q. Jia, Nano Letters, DOI: 10.1021/acs.nanolett.6b02454 (2016).

[11] J. Xie and Y. Xie, Chem. Eur. J. (2015).

[12] T. Wang, X. Wang, Y. Liu, J. Zheng and X. Li, Nano Energy 22 (2016) 111-119.

[13] M. Chhowalla, H. S. Shin, G. Eda, L. J. Li, K. P. Loh and H. Zhang, Nat Chem 5 (2013) 263-275.

[14] Y. Liu, Y. Li, H. Kang, T. Jin and L. Jiao, Mater. Horiz. 3 (2016) 402-421.

[15] W. Zhou, X.-J. Wu, X. Cao, X. Huang, C. Tan, J. Tian, H. Liu, J. Wang and H. Zhang, Energy Environ. Sci. 6 (2013) 2921-2924.

[16] W. Zhou, J. Jia, J. Lu, L. Yang, D. Hou, G. Li and S. Chen, Nano Energy 28 (2016) 29-43.

[17] Y. Zheng, Y. Jiao, Y. Zhu, L. H. Li, Y. Han, Y. Chen, A. Du, M. Jaroniec and S. Z. Qiao, Nat. Commun. 5 (2014).

[18] Y. Zhao, R. Nakamura, K. Kamiya, S. Nakanishi and K. Hashimoto, Nat. Commun. 4 (2013).

[19] E. J. Popczun, J. R. McKone, C. G. Read, A. J. Biacchi, A. M. Wiltrout, N. S. Lewis and R. E. Schaak, J. Am. Chem. Soc. 135 (2013) 9267-9270. 
[20] D. Li, H. Baydoun, C. N. Verani and S. L. Brock, J. Am. Chem. Soc. 138 (2016) 4006-4009.

[21] L.-A. Stern, L. Feng, F. Song and X. Hu, Energy Environ. Sci. 8 (2015) 2347-2351.

[22] J. Tian, Q. Liu, A. M. Asiri and X. Sun, J. Am. Chem. Soc. 136 (2014) 7587-7590.

[23] J. F. Callejas, J. M. McEnaney, C. G. Read, J. C. Crompton, A. J. Biacchi, E. J. Popczun, T. R. Gordon, N. S. Lewis and R. E. Schaak, ACS nano 8 (2014) 11101-11107.

[24] H. F. Liang, A. N. Gandi, D. H. Anjum, X. B. Wang, U. Schwingenschlogl and H. N. Alshareef, Nano Letters 16 (2016) 7718-7725.

[25] J. Li, M. Yan, X. Zhou, Z. Q. Huang, Z. Xia, C. R. Chang, Y. Ma and Y. Qu, Adv. Funct. Mater. 26 (2016) 6785-6796.

[26] Y. W. Tan, H. Wang, P. Liu, Y. H. Shen, C. Cheng, A. Hirata, T. Fujita, Z. Tang and M. W. Chen, Energy Environ. Sci. 9 (2016) 2257-2261.

[27] A. Dutta and N. Pradhan, J. Phys. Chem. Lett. 8 (2016) 144-152.

[28] C. G. Read, J. F. Callejas, C. F. Holder and R. E. Schaak, ACS Appl. Mater. Interfaces 8 (2016) 12798-12803.

[29] Y. P. Zhu, Y. P. Liu, T. Z. Ren and Z. Y. Yuan, Adv. Funct. Mater. 25 (2015) 7337-7347.

[30] O. Tegus, E. Brück, K. Buschow and F. De Boer, Nature 415 (2002) 150-152.

[31] A. C. Colson, C. W. Chen, E. Morosan and K. H. Whitmire, Adv. Funct. Mater. 22 (2012) 1850-1855.

[32] A. P. Leitner, D. E. Schipper, J. H. Chen, A. C. Colson, I. Rusakova, B. K. Rai, E. Morosan and K. H. Whitmire, Chem. Eur. J. 23 (2017) 5565-5572.

[33] A. P. Leitner, J.-H. Chen, D. E. Schipper and K. H. Whitmire, Chem. Mater. 28 (2016) 7066-7071.

[34] J. Li and N. Wu, Catal. Sci. Tech. 5 (2015) 1360-1384.

[35] D. E. Schipper, Z. Zhao, A. P. Leitner, L. Xie, F. Qin, M. K. Alam, S. Chen, D. Wang, Z. Ren and Z. Wang, ACS nano 11 (2017) 4051-4059.

[36] A. C. Colson and K. H. Whitmire, Organometallics 29 (2010) 4611-4618.

[37] Z. Liu, X. Lu, P. Peng, W. Wu, S.-S. Pei, Q. Yu and J. Bao, Phys. Rev. B 82 (2010) 155435.

[38] Q. Yu, L. A. Jauregui, W. Wu, R. Colby, J. Tian, Z. Su, H. Cao, Z. Liu, D. Pandey and D. Wei, Nat. Mater. 10 (2011) 443-449.

[39] A. Larson and R. Von Dreele, Los Alamos National Lab. Rep. No, LA-UR-86-748, 1994.

[40] B. H. Toby, J. Appl Cryst. 34 (2001) 210-213.

[41] C. Powell, Journal of Electron Spectroscopy and Related Phenomena 185 (2012) $1-3$.

[42] V. Di Castro and G. Polzonetti, Journal of Electron Spectroscopy and Related Phenomena 48 (1989) 117-123.

[43] P. E. Blanchard, A. P. Grosvenor, R. G. Cavell and A. Mar, Chem. Mater. 20 (2008) 7081-7088. 
[44] C. Xiang, K. M. Papadantonakis and N. S. Lewis, Mater. Horiz. 3 (2016) 169-173.

[45] M. Tahir, L. Pan, F. Idrees, X. Zhang, L. Wang, J.-J. Zou and Z. L. Wang, Nano Energy 37 (2017) 136-157.

[46] C. D. Wang, J. Jiang, T. Ding, G. H. Chen, W. J. Xu and Q. Yang, Adv Mater Interfaces 3 (2016) 1500454.

[47] D. Li, H. Baydoun, B. Kulikowski and S. L. Brock, Chem. Mater. 29 (2017) 3048-3054.

[48] J. Xie, J. Zhang, S. Li, F. Grote, X. Zhang, H. Zhang, R. Wang, Y. Lei, B. Pan and Y. Xie, J. Am. Chem. Soc. 135 (2013) 17881-17888.

[49] M. A. Lukowski, A. S. Daniel, F. Meng, A. Forticaux, L. Li and S. Jin, J. Am. Chem. Soc. 135 (2013) 10274-10277.

[50] Z. Zhang, B. Lu, J. Hao, W. Yang and J. Tang, Chem. Commun. 50 (2014) 11554-11557.

[51] X. G. Wang, Y. V. Kolen'ko, X. Q. Bao, K. Kovnir and L. F. Liu, Angew. Chem. Int. Ed. 54 (2015) 8188-8192.

[52] J. M. McEnaney, J. C. Crompton, J. F. Callejas, E. J. Popczun, C. G. Read, N. S. Lewis and R. E. Schaak, Chem. Commun. 50 (2014) 11026-11028.

[53] L. Fan, P. F. Liu, X. Yan, L. Gu, Z. Z. Yang, H. G. Yang, S. Qiu and X. Yao, Nat. Commun. 7 (2016).

[54] A. Sivanantham, P. Ganesan and S. Shanmugam, Advanced Functional Materials 26 (2016) 4661-4672.

[55] Y. Wang, C. Xie, D. Liu, X. Huang, J. Huo and S. Wang, (2016).

[56] A. Sivanantham, P. Ganesan and S. Shanmugam, Adv. Funct. Mater. 26 (2016) 4661-4672.

[57] X. Gao, H. Zhang, Q. Li, X. Yu, Z. Hong, X. Zhang, C. Liang and Z. Lin, Angew. Chem. Int. Ed. 55 (2016) 6290-6294.

[58] Y. H. Chang, C. T. Lin, T. Y. Chen, C. L. Hsu, Y. H. Lee, W. Zhang, K. H. Wei and L. J. Li, Adv. Mater. 25 (2013) 756-760.

[59] H.-F. Wang, C. Tang and Q. Zhang, J. Mater. Chem. A 3 (2015) 16183-16189.

[60] E. Paparazzo, Journal of electron spectroscopy and related phenomena 43 (1987) 97-112.

[61] R. Docherty, G. Clydesdale, K. Roberts and P. Bennema, J. Phys. D: Appl. Phys. 24 (1991) 89.

[62] J. K. Nørskov, T. Bligaard, A. Logadottir, J. Kitchin, J. G. Chen, S. Pandelov and U. Stimming, J. Electrochem. Soc. 152 (2005) J23-J26.

[63] Z. Chen, W. Ren, L. Gao, B. Liu, S. Pei and H.-M. Cheng, Nat. Mater. 10 (2011) 424-428. 